\title{
Coronas implantosoportadas: una alternativa de tratamiento restaurador en paciente con bruxismo. Reporte de caso
}

\section{Implant-supported crowns: an alternative for restorative treatment in patient with bruxism. Case report}

\author{
Edwin Alfredo Estevez-Avendaño (iD) ${ }^{1}$, Midian Clara Castillo-Pedraza (iD) ${ }^{2}$, Kelly Johana Rangel-Cantillo (iD) ${ }^{3}$ \\ 1. Universidad del Magdalena. Santa Marta, Colombia. Correo: edwinestevez82@gmail.com - https://orcid.org/0000-0002-7011-9854 \\ 2. Universidad del Magdalena. Santa Marta, Colombia. Correo: midianclar@gmail.com - https://orcid.org/0000-0003-3170-3959 \\ 3. Universidad del Magdalena. Santa Marta, Colombia. Correo: kerac28@gmail.com - https://orcid.org/0000-0002-4488-3808
}

Tipología: Reporte de caso clinico

Para citar este artículo: Estevez-Avendaño EA, Castillo-Pedraza M, Rangel-Cantillo KJ. Coronas implantosoportadas: una alternativa de tratamiento restaurador en paciente con bruxismo. Reporte de caso. Duazary. 2021 septiembre; 18(3 número especial): 100-108. Doi: https://doi.org/10.21676/2389783X.4264

\section{RESUMEN}

Palabras clave: bruxismo; implantes dentales; prótesis dental de soporte implantado.
El bruxismo es un hábito parafuncional que ocurre durante el sueño y/o la vigilia. Siendo más común el bruxismo del sueño, se presenta en el $17 \%$ de la población mundial. Por lo general, cuando es persistente provoca detrimento en la salud oral. No se ha establecido un tratamiento efectivo contra esta parafunción. El bruxismo también se ha considerado como factor de riesgo para la instalación de implantes y se ha asociado con un aumento de complicaciones mecánicas en la rehabilitación protésica. Aunque algunos autores consideran que el bruxismo no afecta la supervivencia del implante, es de suma importancia considerar todos los aspectos relacionados que contribuyan a reducir los efectos de este hábito en rehabilitación implantosoportada. En este estudio, se presenta el caso de un paciente masculino de 58 años, bruxista, rehabilitado sobre implantes dentales con éxito, después de dos años de instalación. Además, se exponen recomendaciones que ayuden a minimizar los efectos nocivos, cuando se diagnostique un paciente bruxista y se pretenda rehabilitar sobre implantes dentales.

\section{ABSTRACT}

Keywords: Bruxism; Dental implants; Dental prosthesis implantsupported.
Bruxism is a parafunctional habit that occurs during sleep and / or wakefulness. Being more common sleep bruxism, which occurs in $17 \%$ of the world population. In general, when it is persistent, it causes detriment to oral health. An effective treatment to stop bruxism does not have been established. Bruxism has also been considered as a risk factor for implants and has been associated with increased mechanical complications in prosthetic rehabilitation. Although some authors consider that bruxism does not affect implant survival, it is extremely important to consider all related aspects that contribute to reducing the effects of this habit in implant-supported rehabilitation. In this study, we present the case of a 58-year-old male patient, bruxist, successfully rehabilitated on dental implants, after two years of evolution. In addition, recommendations are set out to help minimize the harmful effects when a bruxist patient is diagnosed and it is intended to rehabilitate on dental implants. 


\section{INTRODUCCIÓN}

El bruxismo es una actividad muscular anormal, caracterizada por el hábito de rechinar o frotar los dientes, repetida e involuntaria, durante el sueño y/o la vigilia ${ }^{1,2}$. Se presenta en cerca del $5 \%$ de la población mundial como bruxismo de vigilia y en el $17 \%$ como bruxismo del sueño ${ }^{3}$, siendo más frecuente en mujeres ${ }^{3}$.

Aunque la etiología aún está en discusión, un gran número de estudios atribuyen su aparición al estrés, trastornos respiratorios durante el sueño e incluso una mala alimentación ${ }^{4,5}$. En cuanto al diagnóstico, la literatura propone varios métodos. Sin embargo, la evaluación clínica, combinada con el uso de cuestionarios, sigue siendo la mejor opción. Adicionalmente, el uso de electromiografía y polisomnografia suelen ser efectivas ${ }^{6}$.

Actualmente, no existe tratamiento específico que pueda contrarrestar este hábito, aunque varios estudios proponen la combinación farmacológica, psicológica y odontológica ${ }^{7}$. En el caso, donde estos pacientes se pretendan rehabilitar con coronas sobre implante, se han sugerido unas guías clínicas que orientan en el número y dimensión de los implantes, el diseño de la oclusión, los patrones de articulación y la protección de la restauración con una férula dura de estabilización oclusal ${ }^{8}$.

No obstante, hay cierta preocupación cuando se pretende rehabilitar sobre implante un paciente bruxista. Debido a que esta condición provoca sobrecarga en la restauración, pérdida de hueso alveolar y consecuentemente perdida del implante ${ }^{5,9,10}$. En el bruxismo, las fuerzas generadas aumentan las cargas de masticación fisiológica ${ }^{9}$. Por tal motivo, no es inusual que a menudo sea considerada un factor de riesgo para la rehabilitación sobre implantes.

De este modo, pacientes con bruxismo rehabilitados con implantes se ha convertido en un tema de discusión constante, desde hace varias décadas ${ }^{5,11}$. El efecto real del bruxismo sobre las rehabilitaciones con implantes dentales todavía no está bien claro. En este artículo se presenta un caso exitoso de paciente con bruxismo después de dos años de tratamiento con rehabilitación implantosoportada.

\section{REPORTE DE CASO CLÍNICO}

Paciente de sexo masculino, de 58 años, que acude a la Clínica de Operatoria Dental y Estética de la Universidad Nacional de Colombia, sede Bogotá, por presentar prótesis parcial fija desadaptada en zona canino-premolar superior derecha.

En la anamnesis, el paciente refirió tener el hábito de rechinar los dientes. Además, manifestó haber asistido a consulta en múltiples ocasiones por fracturas dentales, desadaptaciones en restauraciones. El paciente no relató enfermedades sistémicas.

El examen clínico extraoral de la articulación temporomandibular (ATM) reveló desviación mandibular hacia la izquierda en apertura y hacia la derecha en cierre mandibular; no presentó ruidos articulares, sintomatología dolorosa en los músculos de la masticación y su apertura oclusal fue normal. En el examen clínico intraoral se observaron facetas de desgaste, lesiones abfractales y resinas desgastadas como se muestra en la Figura 1. Presencia de coronas implantosoportadas en los dientes $24,25,26,36$ y 46 sin alteraciones clínicas visibles, espacio edéntulo en el área del 35 y desadaptación de prótesis parcial fija metalocerámica dentosoportada en zona caninopremolar superior derecha. Por lo tanto, fue necesario incluir en el plan de tratamiento: la confección de coronas individuales dentosoportadas del 13 y 15 , instalación de implantes y posterior confección de coronas implantosoportadas en las zonas del 14 y 35 .
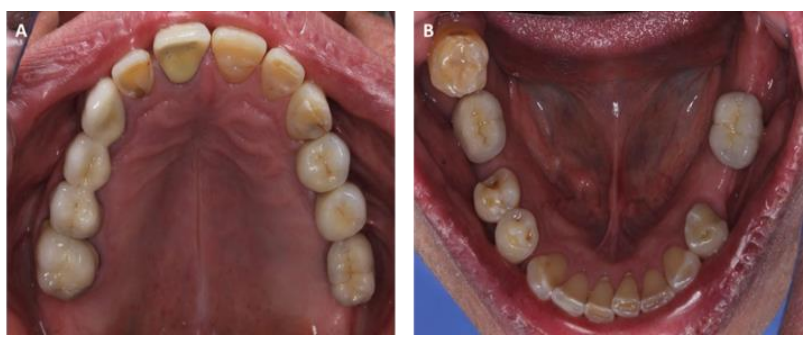

Figura 1. Vista oclusal inicial de las arcadas superior (A) e inferior (B). 
Inicialmente se realizó una junta interdisciplinaria prequirúrgica (periodoncia, rehabilitación oral, operatoria dental y estética), para determinar la posición ideal de los implantes, el procedimiento quirúrgico, las posibles complicaciones, tipo de prótesis a confeccionar, expectativas funcionales y estéticas del paciente según sus necesidades y posibilidades económicas. Se procedió al estudio prostodóntico con la toma de impresiones preliminares de ambas arcadas dentales, la obtención de modelos, el registro de la relación interoclusal, registro bicondilomaxilar con arco facial, montaje de modelos en el articulador, y un encerado diagnóstico para analizar las distintas fuerzas oclusales que incidirían sobre los implantes y así proyectar el resultado estético final.

Posteriormente, se llevó a cabo una terapia de ambientación periodontal y un tratamiento de conducto convencional del 13 para luego cementar un núcleo colado en oro tipo III. Se prosiguió con la repreparación de los pilares 13 y 15 con la finalidad de ajustar una prótesis parcial fija provisional en esta zona, mientras se instalaba el implante del 14.

Del modelo encerado se obtuvo un duplicado para confeccionar dos juegos de férulas guías: uno que se utilizaría para la planificación implantológica en el estudio de la tomografía computarizada del maxilar superior e inferior como se observa en la Figura 2. Y otro juego que serviría de guía quirúrgica para la instalación de cada implante en una posición protésica ideal.

Siguiendo el protocolo de planificación y colocación de implantes según el enfoque de integración sumergida, se procedió a colocar 2 Implantes: el primero en la zona del 14, un implante Dentsply (ANKYLOS, C/X. REF. 3101 0430) de 4,5 mm de diámetro y $11 \mathrm{~mm}$ de largo y el segundo en la zona del 35, un implante Dentsply (ANKYLOS, C/X. REF. 31010425 ) de 4,5 de diámetro y $8 \mathrm{~mm}$ de longitud, como se puede evidenciar en las Figuras $3 \mathrm{~A}$ y $3 \mathrm{~B}$. Finalmente se colocó el tornillo de cicatrización (cover screw) en ambas zonas. zona del 14 y del 35 como se observa en las Figuras $3 C$ y $3 D$ respectivamente. Al siguiente día se colocaron las restauraciones provisionales.

Pasado mes y medio de controles y ajustes de los provisionales a fin de obtener un mejor perfil de emergencia de los tejidos blandos, se programó el inicio de la fase protésica con la toma definitiva de impresiones de los implantes y los dientes a rehabilitar. Las impresiones fueron realizadas utilizando material de Polivinil Siloxano (elite HD+, Zhermack) mediante la técnica de cubeta cerrada para el maxilar superior (coping de impresión ANKYLOS/X Ref. 31047044 y análogo Ref. 3104 7012) y la técnica de cubeta abierta para el maxilar inferior (coping de impresión ANKYLOS/X Ref. 3104 7040 y análogo Ref. 3104 7012).

Finalmente, se eligieron los pilares para los implantes (Rectos Prefabricados ANKYLOS Regular/X (indexado) Ref. $31024110 \mathrm{GH}$ 0,75) y las coronas individuales metal-cerámicas implantosoportadas y dentosoportadas se confeccionaron y cementaron en ambos cuadrantes como se muestra en la Figura 4.

Se le indicó al paciente el uso de una placa oclusal protectora teniendo en cuenta su diagnóstico de bruxismo y se programaron controles clínicos y radiográficos a los 6 meses, 1 y 2 años después del inicio del tratamiento, para observar si el bruxismo influyó en la sobrevivencia de los implantes, los tejidos periimplantares y estética de la restauración, siguiendo los criterios para determinación del éxito de los implantes, sugerido por Albrektsson et a ${ }^{12}$.

Durante la evaluación clínica en los controles a los 6 meses, 1 y 2 años, no se observó movilidad de los implantes, ni dolor por palpación o percusión. Tampoco se notaron alteraciones o fracturas en las coronas implantosoportadas como se evidencia en la Figura 5. Así, el tratamiento aplicado condujo a resultados funcionales y estéticos satisfactorios tanto para el paciente como para el equipo de trabajo.

Después de cinco meses de cicatrización, se consiguió la osteointegración de los implantes. De esta manera, se dio inicio a la segunda fase quirúrgica, en la cual se posicionaron los pilares provisionales en los implantes correspondientes a la 


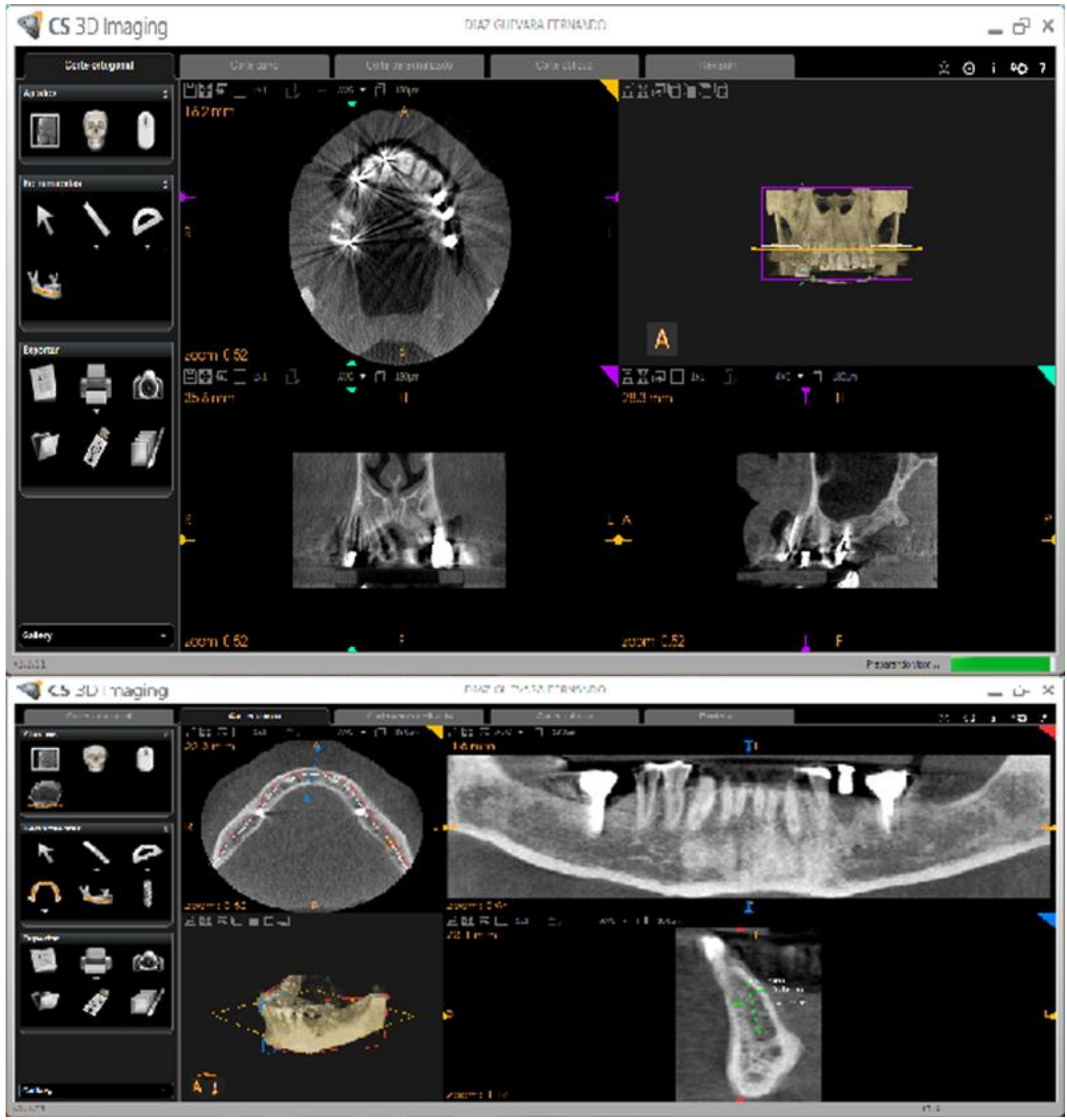

Figura 2. Imágenes diagnosticas tomográficas de la zona edéntula del 14 (A) y 35 (B), utilizando el Software 3D-Imaging. 

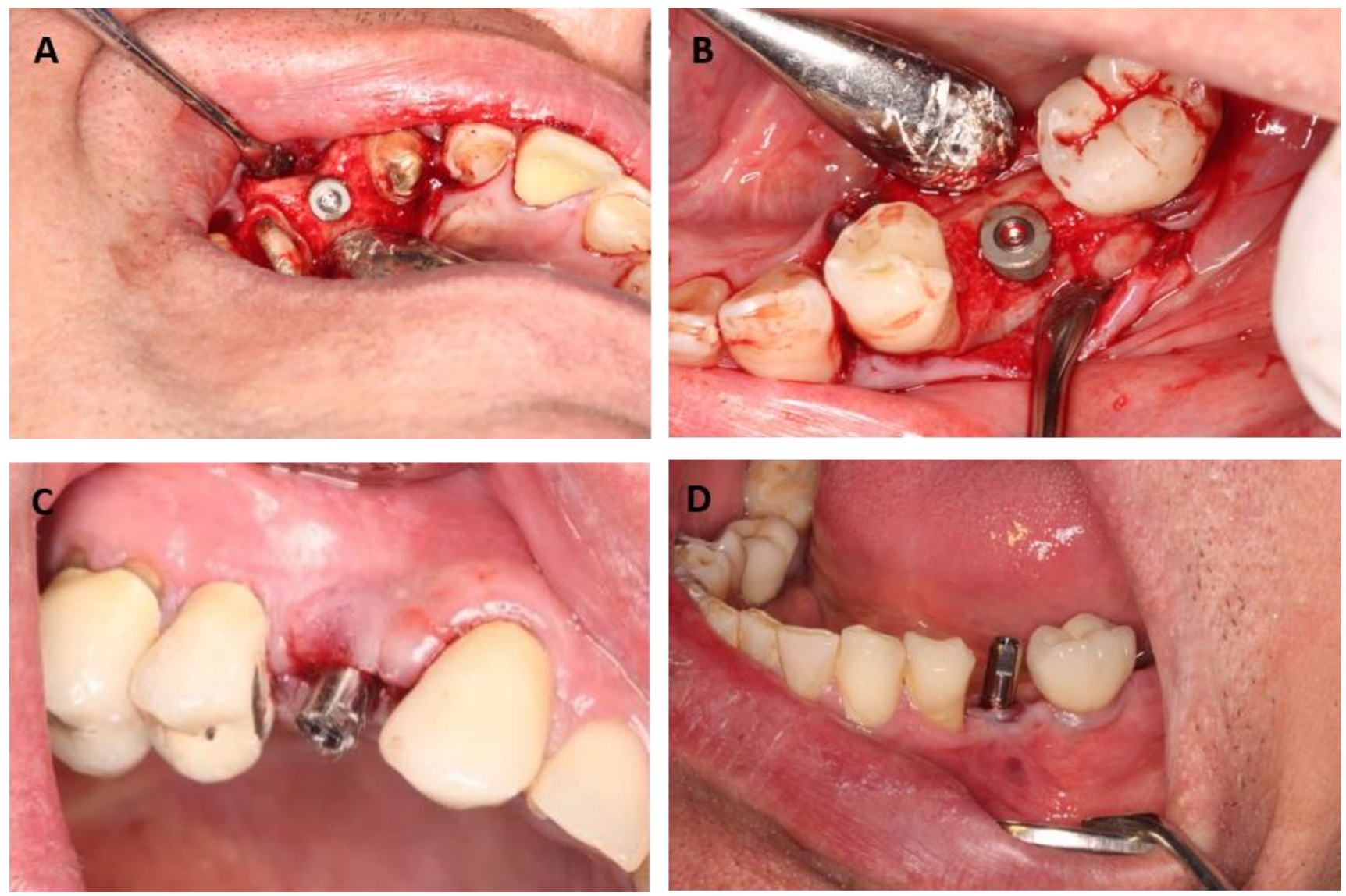

Figura 3. Primera fase quirúrgica: instalación de los implantes correspondiente a la zona del 14 (A) y 35 (B). Segunda fase quirúrgica: instalación de los pilares provisionales en los implantes 14 (C) y 35 (D).
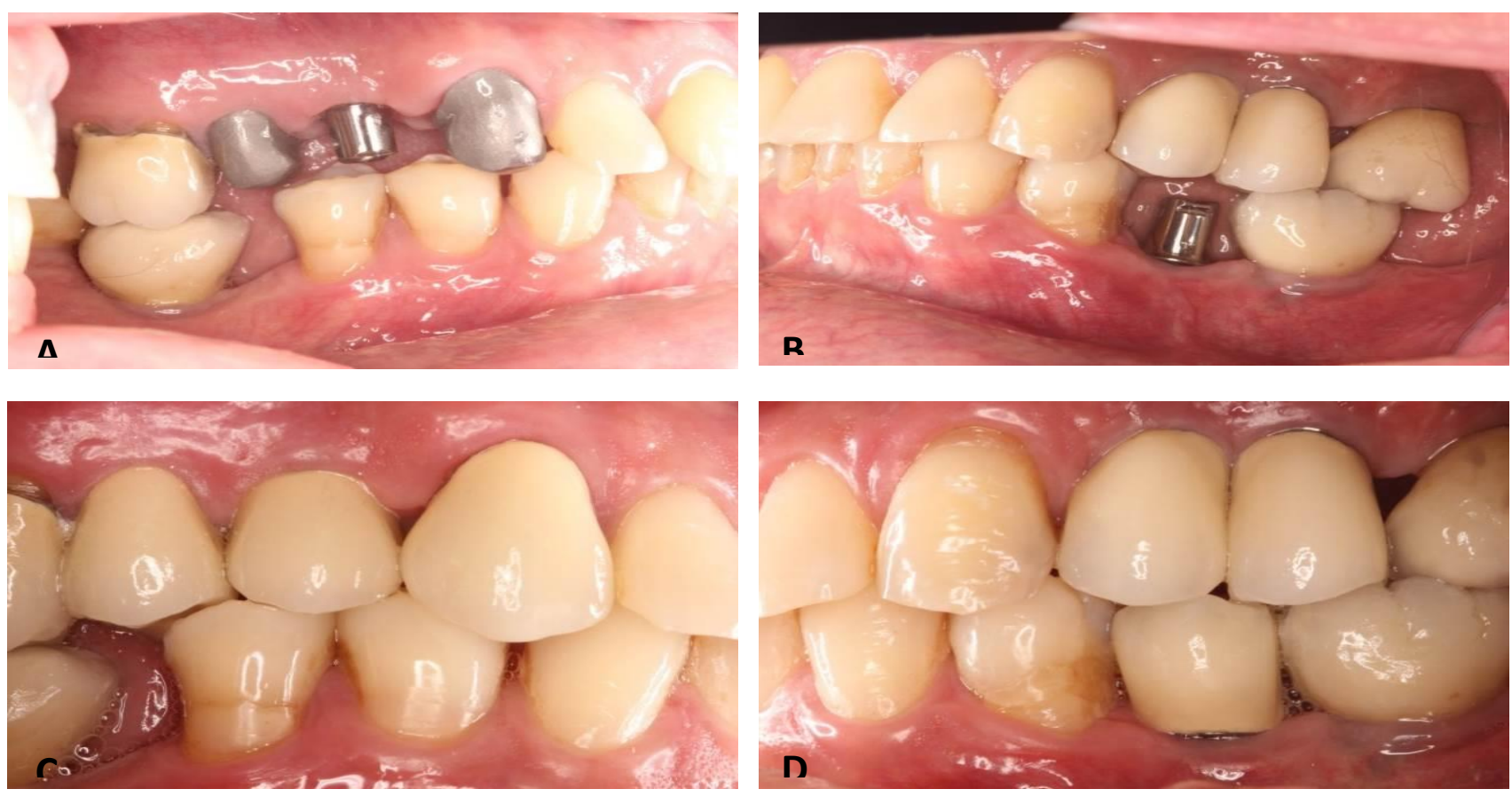

Figura 4. Prueba de cofias metálicas dentosoportadas del 13 y 15, pilares rectos prefabricados implantosopotados del 14 (A) y 35 (B). Cementación de coronas individuales metalocerámicas dentosportadas del 13 y 15 e implantosoportadas del 14 (C) y 35 (D). 

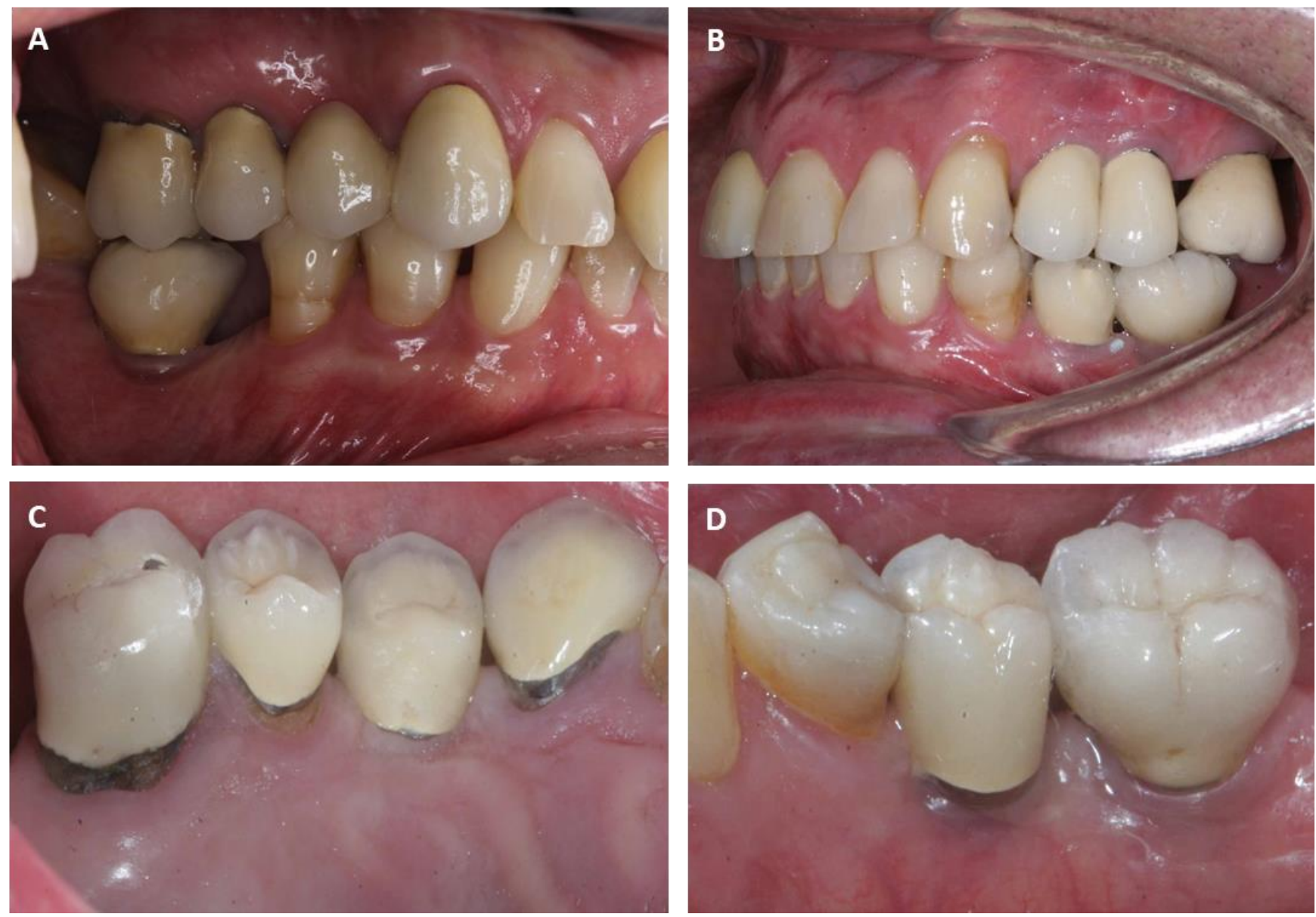

Figura 5. Examen clínico intraoral después de dos años de instalación de la prótesis implantosoportadas. Vista vestibular de las coronas del 13,14, 15 (A) y del 35 (B). Vista palatina de las coronas del 13, 14, 15 (C) y del 35 (D).

Radiográficamente, se percibe que después de 2 años de instalación del implante 14 no hubo pérdida ósea aparente y en el implante 35 se observa por distal una disminución ósea vertical menor de 1,5 $\mathrm{mm}$ en comparación con la radiografía de instalación como se muestra en la Figura 6. Los valores observados se encuentran dentro de los criterios de éxito del implante a término temprano ${ }^{12,13}$.

\section{Declaración sobre aspectos éticos}

Los autores declaran que los procedimientos cumplen los principios éticos de la Declaración de Helsinki y la Resolución 8430 de 1993 del Ministerio de Salud de Colombia. El paciente autorizó por medio del consentimiento informado la utilización de fotos e información de la atención clínica. La información es custodiada acorde a la Ley Estatutaria 1581 de 2012.
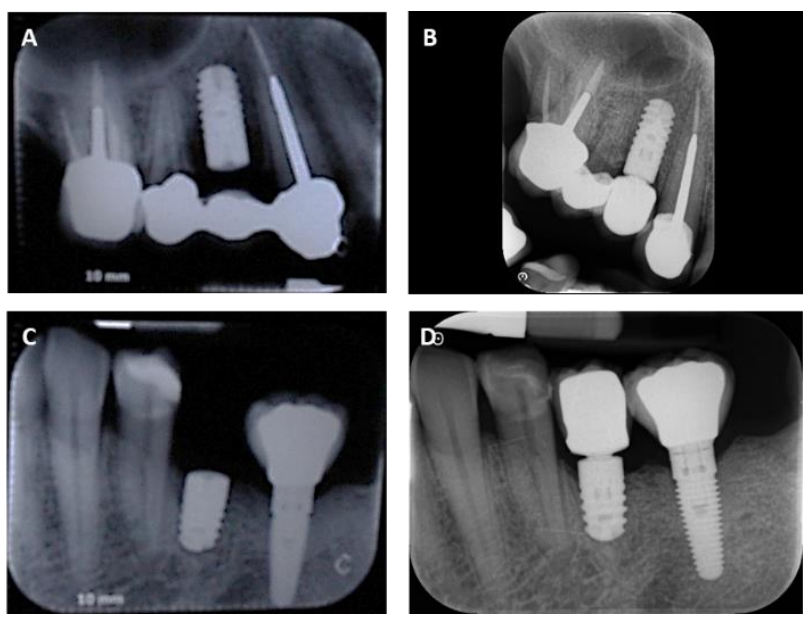

Figura 6. Imagen radigrafica inicial del implante correspondiente a la zona del 14 (A) y después de 2 años de instalación (B). Imagen radigrafica inicial del implante correspondiente a la zona del 35 (C) y después de 2 años de instalación (D). 


\section{DISCUSIÓN}

Los implantes dentales generalmente son considerados la primera elección para rehabilitar pacientes con edentulismo parcial o total. Sin embargo, aunque la literatura ha establecido que la rehabilitación sobre implante presenta alta tasa de éxito clínico y longevidad ${ }^{14-16}$, enfermedades sistémicas como diabetes, osteoporosis, periodontitis, pacientes con tratamientos radioterápicos antes o después de la instalación del implante y pacientes fumadores, pueden llevar al fracaso de los implantes dentales ${ }^{17-19}$.

Diversos autores coinciden en que el bruxismo debe ser considerado un factor de riesgo, debido a que pone en peligro el éxito de la rehabilitación basada en implantes ${ }^{5,10,11}$. A pesar de esto, no es absolutamente claro el efecto del bruxismo sobre la supervivencia del implante. En general, las sugerencias formuladas en este campo parecen estar basadas en opiniones de expertos y no en la evidencia científica ${ }^{8,20}$.

Chrcanovic et $a l^{21}$ en un estudio comparativo con 98 pacientes, analizaron las complicaciones del tratamiento con implantes dentales en pacientes con o sin bruxismo. En este estudio se señala que, en pacientes bruxistas puede aumentar la falla del implante y en las restauraciones. Sin embargo, no deja claro el tiempo promedio transcurrido para la reevaluación clínica de los pacientes. Manfredini et $a l^{5}$ resaltan que el bruxismo no debería ser considerado como una contraindicación en las rehabilitaciones sobre implantes, debido que no ofrecen complicaciones biologicas considerables, pero si sugiere que puede ofrecer complicaciones mecánicas en la restauración. De Angelis et $a{ }^{22}$, sugieren que el bruxismo es un factor de riesgo tardío considerable, sobre la rehabilitación implantosoportada en pacientes que no han superado esta condición.

En particular, es difícil comparar los resultados de los diversos estudios, debido a las diferencias metodológicas, las características de las muestras investigadas y los métodos de diagnóstico utilizados para detectar el bruxismo o para evaluar el fracaso del implante ${ }^{20}$.
En este estudio, se demostró que el tratamiento con implantes en pacientes con bruxismo es una alternativa compatible por lo menos a corto plazo. Para esto, se realizaron evaluaciones clínicas y radiográficas, en diferentes periodos de tiempos, y no se evidenció influencia de alteraciones provocadas por el aumento de fuerza, pérdida de crestal ósea temprana, aflojamiento del pilar o del tornillo protésico, fractura de porcelana o fractura del componente ${ }^{12,14,23}$.

Asimismo, se notó que las coronas dentosoportadas tampoco sufrieron afecciones aparentes por estas sobre cargas. Esto podría obedecer a un mejor autocontrol del estrés por parte del paciente, toda vez que el estrés es considerado un factor etiológico asociado al bruxismo ${ }^{4}$. Además del que paciente asistió regularmente a todos sus controles.

Si bien varios estudios ${ }^{11,17,19,21,22}$ han evaluado la sobrevivencia del implante en pacientes con bruxismo, los resultados no han sido consistente. Ante la falta de un acuerdo, se propone establecer una serie de medidas clínicas que minimicen los efectos nocivos cuando se diagnostique un paciente bruxista y se pretenda rehabilitar con implantes dentales. Algunas de estas medidas podrían ser:

i. Elaboración de un adecuado diagnostico utilizando los recursos y tecnologías actuales.

ii. Diagnosticar cualquier alteración oclusal existente, que deba ser tratada antes de la ejecución del tratamiento, tales como prematuridades, trauma oclusal, interferencias oclusales.

iii. En edéntulos parciales: Emplear un mayor número de implantes y de mayor diámetro en la medida de lo posible. Reducir la extensión de las arcadas aplicando conceptos de "arcada corta" para alejar las restauraciones del eje de bisagra, donde se ejercen fuerzas oclusales mayores. Reducir, si posible, caras oclusales de los molares.

iv. En pacientes con denticiones muy desgastadas, será importante conseguir una adecuada guía anterior. En caso de tener que emplear implantes de diámetros reducidos, considerar la elección de nuevos materiales de mayor resistencia. 
v. Emplear férulas de descarga de uso nocturno como método complementario a la rehabilitación de estos pacientes.

Además, se propone rehabilitar sobre implantes los pacientes que presenten bruxismo y este no esté acompañado con otra condición considerada como factor de riesgo. Es necesario también, el acompañamiento constante de estos pacientes para tratar de neutralizar o eliminar esta condición. Una buena propuesta sería la utilización de toxina botulínica $^{24}$ como tratamiento del bruxismo, que mejora los niveles de dolor y la fuerza oclusal máxima, aún en los casos graves de la condición.

\section{CONCLUSIÓN}

La rehabilitación con implantes osteointegrados pueden ser una opción exitosa en pacientes con bruxismo a término temprano, una vez se realicen los ajustes oclusales necesarios y se lleven a consideración las medidas clínicas que minimicen los detrimentos sobre la restauración y el periodonto. Sin embargo, se hace necesario un abordaje científico, que permita explicar con veracidad, el efecto del bruxismo sobre el implante dental.

\section{DECLARACIÓN SOBRE CONFLICTOS DE INTERÉS}

Los autores informan que no existe conflicto de interés y el artículo no es financiado o apoyadas por ninguna beca de investigación.

\section{CONTRIBUCIÓN DE LOS AUTORES}

Primer autor: trabajo de campo, redacción y contextualización.

Segundo autor: redacción, contextualización y asesor de documentación.

Tercer autor: redacción y asesor de documentación.

\section{REFERENCIAS BIBLIOGRÁFICAS}

1. Lavigne GJ, Rompre PH, Montplaisir JY. Sleep Bruxism: Validity of Clinical Research Diagnostic Criteria in a Controlled Polysomnographic Study. J
Dent Res. 1996 Jan 8;75(1):546-52. Doi: https://doi.org/10.1177/00220345960750010601

2. Lobbezoo F, Ahlberg J, Glaros AG, Kato T, Koyano $\mathrm{K}$, Lavigne GJ, et al. Bruxism defined and graded: An international consensus. J Oral Rehabil. 2013;40(1):2-4.

Doi: https://doi.org/10.1111/joor.12011

3. Wetselaar P, Vermaire EJH, Lobbezoo F, Schuller AA. The prevalence of awake bruxism and sleep bruxism in the Dutch adult population. J Oral Rehabil. 2019;46(7):617-23. Doi: https://doi.org/10.1111/joor.12787

4. Johansson A, Omar R, Carlsson GE. Bruxism and prosthetic treatment: A critical review. J Prosthodont Res. 2011;55(3):127-36. Doi: https://doi.org/10.1016/j.jpor.2011.02.004

5. Manfredini D, Poggio CE, Lobbezoo F. Is Bruxism a Risk Factor for Dental Implants? A Systematic Review of the Literature. Clin Implant Dent Relat Res. 2014;16(3):460-9. Doi: https://doi.org/10.1111/cid.12015

6. Lobbezoo F, Ahlberg J, Manfredini D, Winocur E. Are bruxism and the bite causally related? J Oral Rehabil. 2012;39(7):489-501. Doi: https://doi.org/10.1111/j.1365-2842.2012.02298.x

7. Macedo $C R$, Silva $A B$, Machado MA, Saconato $H$, Prado GF. Occlusal splints for treating sleep bruxism (tooth grinding). Cochrane Database Syst Rev. 2007;(4):CD005514. Doi: https://doi.org/10.1002/14651858.CD005514.pub2

8. Lobbezoo F, Van Der Zaag J, Naeije M. Bruxism: Its multiple causes and its effects on dental implants An updated review. J Oral Rehabil. 2006;33(4):293300. Doi: https://doi.org/10.1111/j.13652842.2006.01609.x

9. Chrcanovic BR, Albrektsson T, Wennerberg A. Bruxism and dental implants: A meta-analysis. Implant Dent. 2015;24(5):505-16. Doi: https://doi.org/10.1097/ID.0000000000000298 
10. Zhou Y, Gao J, Luo L, Wang Y. Does Bruxism Contribute to Dental Implant Failure? A Systematic Review and Meta-Analysis. Clin Implant Dent Relat Res. 2016;18(2):410-20. Doi: https://doi.org/10.1111/cid.12300

11. Zortuk M, Kilic E, Yildiz P, Leblebicioglu I. Effect of parafunctional force on dental implant treatment in bruxism: A case report (two year results). J Int Dent Med Res. 2011;4(1):25-9. Disponible en: ktodermaldisplazi.com/journal/Journal2011/Nol4_ No1/6_D1086_Mustafa_Zortuk.pdf

12. Albrektsson $T$, Zarb G, Worthington P EA. The long-term efficacy of currently used dental implants: a review and proposed criteria of success. Int J Oral Maxillofac Implant. 1986;1(1):11-25. Disponible en: http://www.quintpub.com/journals/omi/abstract.p hp?article_id=11202\#.YMlfcqhKjIV

13. Misch $\mathrm{CE}$, Morton $\mathrm{P}$, Wang $\mathrm{HL}$ et al. Implant success, survival and failure: The International Congress of Oral Implantologist (ICOI) Pisa Consensus Conference. Implant Dent. 2008;17(1):515. Doi:

https://doi.org/10.1097/ID.0b013e3181676059

14. Naert I, Koutsikakis G, Duyck J, Quirynen M, Jacobs $R$ van SD. Biologic outcome of implantsupported restorations in the treatment of partial edentulism. Part 2: a longitudinal radiographic study. Clin Oral Implant Res. 2002;13(4):390-395. Doi: $\quad$ https://doi.org/10.1034/j.16000501.2002.130407.x

15. Lekholm U, Gunne J, Henry P, Higuchi K, Lindén $U$, Bergström $C$ van SD. Survival of the Brånemark implant in partially edentulous jaws: a 10-year prospective multicenter study. Int J Oral Maxillofac Implant. 1999;14(5):639-45. Disponible en: http://medlib.yu.ac.kr/eur_j_oph/ijom/IJOMI/ijomi _14_639.pdf

16. Rameh S, Menhall A YR. Key factors influencing short implant success. Oral Maxillofac Surg. 2020;24(3):263-75. Doi: https://doi.org/10.1007/s10006-020-00841-y

17. Kullar AS, Miller CS. Are There Contraindications for Placing Dental Implants? Dent Clin North Am.
2019;63(3):345-62.

Doi: https://doi.org/10.1016/j.cden.2019.02.004

18. Chen H, Liu N, Xu X, Qu X LES. Smoking, radiotherapy, diabetes and osteoporosis as risk factors for dental implant failure: a meta-analysis. PLoS One. 2013;8(8):e71955. Doi: https://doi.org/10.1371/journal.pone.0071955

19. Klokkevold PR, Han TJ. How do smoking, diabetes, and periodontitis affect outcomes of implant treatment? Int J Oral Maxillofac Implant. 2007;22:173-202.

Doi: https://doi.org/10.1038/bdj.2007.841

20. Manfredini D, Bucci MB, Sabattini VB LF. Bruxism: overview of current knowledge and suggestions for dental implants planning. Cranio. 2011;24(4):3014-312.

Doi: https://doi.org/10.1179/crn.2011.045

21. Chrcanovic BR, Kisch J, Albrektsson T WA. Bruxism and dental implant treatment complications: a retrospective comparative study of 98 bruxer patients and a matched group. Clin Oral Implant Res. 2017;28(7):e1-9. Doi: https://doi.org/10.1111/clr.12844

22. De Angelis F, Papi P, Mencio F, Rosella D, Di Carlo $S$ PG. Implant survival and success rates in patients with risk factors: results from a long-term retrospective study with a 10 to 18 . Eur Rev Med Pharmacol Sci. 2017;21(3):433-7. Disponible en: https://www.europeanreview.org/wp/wpcontent/uploads/433-437-Long-term-follow-upanalysis-of-implant-patients-with-risk-factors.pdf

23. CE M. Dental implant prosthetics [Internet]. 2 edition. Elsevier Mosby. 2015. 1008 p. Available from: https://www.elsevier.com/books/dentalimplant-prosthetics/misch/978-0-323-07845-0

24. Fernández-Núñez T, Amghar-Maach S G-EC. Efficacy of botulinum toxin in the treatment of bruxism: Systematic review. Med Oral Patol Oral Cir Bucal. 2019;24(4):e416-24. Doi: https://doi.org/10.4317/medoral.22923 